V. S. LETOCHOW

Laserspektroskople

DIERCK-EKKEHARD LIEBSCHER

Relativitätstheorie

mit Zirkel und Lineal

Wolfgang Mrining

Digitalrechner

in der elektronischen Meßtechnik

Teil 1: MeBmethodik

Teil 2: Gerätetechnik

und Anwendungen

L. I. Miroschnitschenko

Kosmische Strahlen

im interplanetaren Raum

PkTkR PAUfler

Gustav E. R. Schulze

Physikalische Grundlagen

mechanischer Festkörpereigenschaften

Teil I und II

ULRICH RÖSEBERG

Quantenmechanik und Philosophie

Albrecht Rost

Messung

dielektrischer Stoffelgenschaften

J. V. SACKOV

Wahrscheinlichkeit und Struktur

E. M. SAWIZKI

Perspektiven der Metallforschung

Wolfgang SCHÄFeR

Theoretische Grundlagen

der Stabilität technischer Systeme

ERNST SCHMUTZER

Symmetrien und Erhaltungssätze

der Physik

VOLKMAR SCHURICHT

Kernexplosionen für friediliche $\mathrm{Z}$ wecke

NORBERT SIEBER

HANS-PETER LEIDHOLD

Einführung in die Datenverarbeitung
GERHARD WUNSOH

Zellulare Systeme

Festkörperphysik

Entwicklungstendenzen und

Anwendungsmöglichkeiten

HORST MELCHER

Albert Einstein wider Vorurtelle

und Denkgewohnheiten

Die Schöpter der physikalischen Optik

Eine Artikelsammlung

HANS-GeORe SOHÖPF

Von Kirchhoff bis Planck

RENATE WAHSNER

Mensch und Kosmos

Die copernicanische Wende

HeLmUT Friemel / Joser Brock

Grundlagen der Immunologie

EBERHARD HOFMANN

Funktionelle Biochemie des Menschen

Band 1 und 2

LOTHAR JÃGER

Grundlagen

der Klinischen Immunologte

KARLHEINZ LOHS

Dieter MARTINeTZ

Entgiftung - Mittel, Methoden

und Probleme

JOACHIM NitschmanN

Entwlcklung bei Mensch und Tier

STEPHAN SCHNITZLER

Pharmakologische Aspekte

von Immunreaktionen

DIBTER SPAAR

HELMUT KLEINHEMPEL

HANS JOACHIM MULLER

KLAUS NAUMANN

Bakteriosen der Kulturpflanzen 
EberHard TrUSCher

Pharmakognosle

Teil I-III

Michael Theile

SIEGRRIED SCHERNECK

Zellgenetik

HEINRICH BREMER

KLAUS-PETER WendlandT

Heterogene Katalyse

PETER BIRNER

HANS-JÖRG HOFMANN

CORNELIUS WeIsS

MO-theoretische Methoden

in der organischen Chemie

GUNTTER EPPERT

Einftihrung

in die Schnelle Flüssigchromatographle

Gerhard Geiseler / Heinz Seider

Die Wasserstoffbrückenblndung

HaLMUT HRAPIA

Einführung in die Chromatographie

HANS LUPPA

Grundlagen der Histochemie

Teil I und II

BURKart PHILIPP

GRRHARD REINISOH

Grundlagen

der makromolekularen Chemie

HORST REMANE / RAINER HERZSCHUH Massenspektrometrle

in der organischen Chemie

ADOLF ZSCHUNER

Kernmagnetische

Resonanzspektroskopie

in der organischen Chemie
Vorschau

auf die nächsten Bände:

HEINRICH ARNOLD

Physikalische Chemie der Halbleiter

HORST-HEINO VON BORZESZKOWVKI

RENATE WAHSNER

Newton und Voltaire

WERNER DÖPKE

Dynamische Aspekte der Stereochemie organischer Verbindungen

GOTTRRIED FRITZSCHE

Entwurf aktiver Analogsysteme

Netzwerke III

FALKO H. HERRMANN

MARINA HERRMANN

Das Hämoglobin des Menschen

GERHARD JACEISCH

Johann Heinrich Lamberts

"Cosmologische Briefe" mit Beiträgen zur Frühgeschichte der Kosmologie

Das Neutron

Eine Artikelsammlung

Volker Noltad

Semi-Markovsche Prozesse

ROBERT ROMPE

HANS-JURGEN TREDER

Über Physik -

Studien zu Ihrer Stellung

in Wissenschaft und Gesellschaft

RAINER SINZ

Chronopsychophysiologie,

Chronobiologie und Chronomedizin

HUBERTUS STOL?

Supraleitung 


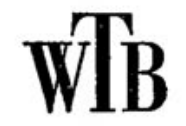

BAND 191

\author{
Dieter Leuschner
}

\title{
Grundbegriffe der Thermodynamik
}

Mit 17 Abbildungen und 6 Tabellen 


\section{Reihe MATHEMATIK UND PHYSIK}

\section{Herausgeber :}

Prof. Dr. phil. habil. w. Holzmüller, Leipzig

Prof. Dr. phil. habil. A. Lösche, Leipzig

Prof Dr. phil. habil. H. Reichardt, Berlin

Prof. Dr. rer. nat. habil. H.-J. Treder, Potsdam

Verfasser :

Dr. rer. nat. Dieter Leuschner

Akademie der Landwirtschaftswissenschaften der DDR

Erschienen im Akademie-Verlag, 108 Berlin, Leipziger Str. 3-4

(c) Akademie-Verlag Berlin 1979

Lizenznummer: $202 \cdot 100 / 435 / 79$

Herstellung: VEB Druckhaus „Maxim Gorki“, 74 Altenburg

Bestellnummer: 7623408 (7191) · LSV 1144

Printed in GDR

DDR 8,- M 